\title{
Linear-optical processing cannot increase photon efficiency
}

\author{
D. W. Berry ${ }^{1}$ and A. I. Lvovsky ${ }^{2}$ \\ ${ }^{1}$ Institute for Quantum Computing, University of Waterloo, Ontario N2L 3G1, Canada \\ ${ }^{2}$ Institute for Quantum Information Science, University of Calgary, Alberta T2N 1N4, Canada
}

\begin{abstract}
We answer the question whether linear-optical processing of the states produced by one or multiple imperfect single-photon sources can improve the single-photon fidelity. This processing can include arbitrary interferometers, coherent states, feedforward, and conditioning on results of detections. We show that without introducing multiphoton components, the single-photon fraction in any of the single-mode states resulting from such processing cannot be made to exceed the efficiency of the best available photon source. If multiphoton components are allowed, the single-photon fidelity cannot be increased beyond $1 / 2$. We propose a natural general definition of the quantum-optical state efficiency, and show that it cannot increase under linear-optical processing.
\end{abstract}

Optical implementation of quantum information processing and communication employs the single-photon state as one of its primary resources [1]. There exist a variety of methods to produce this state, both of heralded and on-demand nature [1, 2]. However, no single-photon source is perfect. While many single-photon sources are able to effectively suppress multiphoton components from the output, the produced state typically has a significant admixture of vacuum. In other words, the quantum state of light generated by a typical single-photon source can be approximately written in the photon number basis as

$$
\hat{\rho}=(1-p)|0\rangle\langle 0|+p| 1\rangle\langle 1|,
$$

where $p$ is the efficiency of the source. In the remainder of the paper, we call state (11) the inefficient single photon state (ISPS).

In this work, we are investigating possibilities to enhance the efficiency of an ISPS using linear optical (LO) processing. This processing includes arbitrary operations by means of LO elements (mirrors, beam splitters, etc.), destructive measurements as well as modifications of the LO circuit (feedforward) or postselection based on measurement results. The efficiency of the output ISPS is evaluated for the remaining undetected modes after postselection.

LO processing is attractive because linear optical elements and quantum optical detectors are widely available, inexpensive and versatile; furthermore, they can be integrated in a waveguide circuit [3] . Recent investigations have shown that many tasks of quantum information technology can be accomplished by means of LO processing. A well-known example is an efficient linear optical scheme for quantum computation [4]. Furthermore, corrections of many types of errors, including those caused by photon loss, can be accomplished using such processing $[5]$. This naturally raises the question of whether LO processing can be employed to correct for photon loss itself, thus leading to a single photon source with greater efficiency.

We find the limitations on using LO processing to improve photon sources. We give two main results, resolving long-standing conjectures:
1. If no two or higher photon number components are allowed in the output mode (which is an essential requirement for many applications, such as quantum cryptography), the probability of the singlephoton component in the output cannot be higher than the efficiency of the best single-photon source at the input, $p_{\max }$.

2. If we do allow multiphoton components in the output mode, the single photon weight therein cannot exceed the greater of $p_{\max }$ and $1 / 2$.

Both these statements are general in that they do not impose any restriction on the number of photon sources available, the configuration of the LO scheme or destructive measurements involved.

These results were hypothesized in Refs. [6, 7], which provided numerical evidence and gave proofs in some special cases. Tightness of these results has also been demonstrated. Refs. 6, 8] provided a scheme that can increase the single-photon probability, provided multiphoton components are allowed in the output. This scheme worked if and only if the initial single-photon probability was less than $1 / 2$. In a related work [9], it was shown that if there is initial coherence between the vacuum and singlephoton components, then the single-photon probability can be increased, but at the expense of the coherence. For these partially-mixed states a generalized efficiency was defined, which cannot increase through LO processing of a single source.

One restriction on the schemes we study is that the measurements are destructive. If one could perform a quantum nondestructive (nondemolition) measurement of the number of photons, then postselecting on detection of a single photon would improve the efficiency. However, such measurements require nonlinearity [10], and are not performed by standard photodetectors. It is possible to achieve an effective nondemolition measurement using linear optics and destructive measurements provided that perfect single photons are given as a resource [11]. However, we cannot make use of this possibility because we require that all available single-photon sources are imperfect with quantum efficiency no higher than $p_{\max }$. 
Aside from the above restriction, our results are valid for arbitrary generalized quantum measurements. This includes the case when some of the modes are not measured at all, thus accounting for optical losses or imperfect mode matching on beam splitters.

In general, LO processing schemes can involve feedforward, i.e. LO operations that are controlled by the results of measurements. This is used, for example, in schemes for linear optical quantum computation [5]. Typically, the controlled operations are adjusted before they act on the photons. A scheme with such feedforward can however be replaced by a scheme that is immediately prepared in its final configuration corresponding to the set of measurement results that give the largest singlephoton probability at the output. If postselection on this set of results is employed, the single-photon probability under this fixed scheme will be at least as high as under the scheme with feedforward. Therefore we can without loss of generality eliminate feedforward from our future analysis.

In view of the above, any LO processing scheme can be converted to the form depicted in Fig. 1 [6]. Multiple ISPSs $\hat{\rho}_{1}, \ldots, \hat{\rho}_{M}$ with efficiencies $p_{1}, \ldots, p_{M}$, are combined in a linear interferometer. For added generality, we also allow coherent states (which are readily available from a laser) as inputs. This includes vacuum states as coherent states with amplitude zero. All the interferometer outputs except one are then subjected to a measurement. Conditioned on a particular result of this measurement, we analyze the state in the remaining mode.

We begin our argument by redrawing our scheme as shown in Fig. 2. Because the efficiencies of all initial ISPSs are not greater than $p_{\max }$, they can be interpreted as ISPSs $\hat{\rho}_{i}^{\prime}$ of efficiency $p_{i} / p_{\max }$ that have propagated through optical attenuators of transmissivity $p_{\max }$. The coherent states $\left|\alpha_{i}\right\rangle$ can be interpreted as coherent states

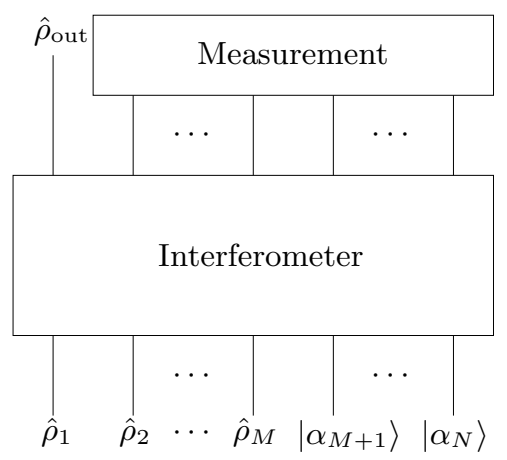

FIG. 1: A general interferometer for processing singlephoton sources. The single-photon sources are the $M$ states $\hat{\rho}_{1}, \ldots, \hat{\rho}_{M}$. In addition, coherent states may be allowed as inputs. The modes pass through a general interferometer, and all but one of the output modes are detected via a measurement. of amplitudes $\alpha_{i}^{\prime}=\alpha_{i} / \sqrt{p_{\max }}$ that have been similarly attenuated.

Now the following observation can be made. The set of attenuators can be commuted with the interferometer without affecting the multimode optical state before the measurement. In other words, the scheme in Fig. 2 is equivalent to that in Fig. 3. To our knowledge, this observation has been made for the first time by Varnava et al. 12] for a specific interferometer configuration. Below, we offer a general proof for an arbitrary LO process.

A multimode state propagating through an attenuator (with equal loss in all the modes) can be described as fictitious time evolution [13]:

$$
\frac{d \hat{\rho}}{d t}=\kappa[\hat{A}(\hat{\rho})-\hat{N}(\hat{\rho})]
$$

where

$$
\hat{A}(\hat{\rho})=\sum_{k} \hat{a}_{k} \hat{\rho} \hat{a}_{k}^{\dagger}
$$

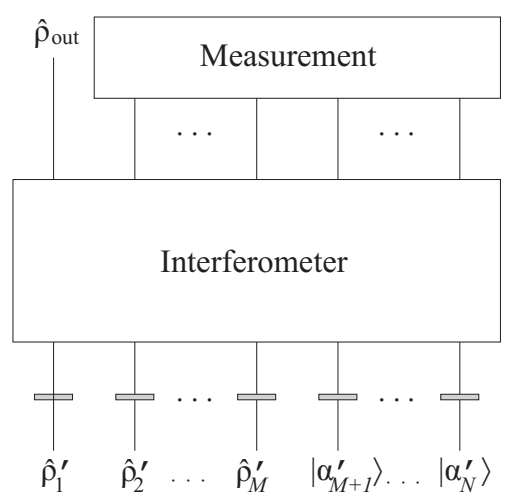

FIG. 2: An equivalent scheme to Fig. 1. All interferometer inputs of Fig. 1 are interpreted as optical states with a higher single-photon probabilities (for inefficient photon sources) or higher amplitudes (for coherent states) that have propagated through identical attenuators (grey boxes) of transmissivity $p_{\max }$.

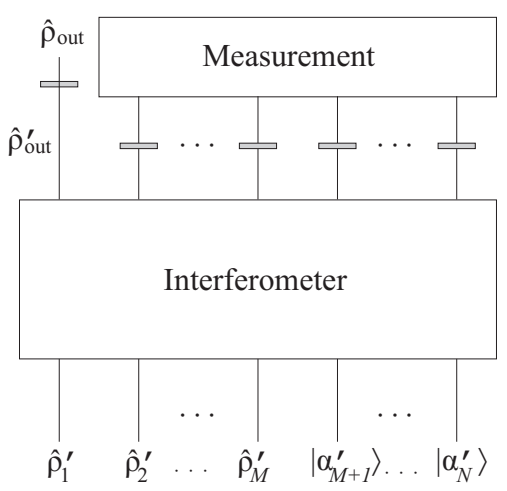

FIG. 3: An equivalent scheme to Fig. 2. The interferometer and attenuators can be interchanged, as shown in the text. 


$$
\hat{N}(\hat{\rho})=\sum_{k}\left(\hat{a}_{k}^{\dagger} \hat{a}_{k} \hat{\rho}+\hat{\rho} \hat{a}_{k}^{\dagger} \hat{a}_{k}\right) / 2,
$$

are the Lindbladian superoperators. Summation is performed over all optical modes with annihilation operators $\hat{a}_{k}, \kappa$ is the loss coefficient such that $p_{\max }=e^{-\kappa t_{0}}$, and $t_{0}$ is the fictitious time during which the loss channel is applied. In writing Eq. (2) we have used the fact that the attenuation is the same in all the modes.

The action of the interferometer can be written in the Heisenberg picture as a unitary transformation between the annihilation operators of the input $\hat{a}_{k}$ and output $\hat{b}_{k}$ modes:

$$
\hat{a}_{j}=\sum_{k} U_{j k} \hat{b}_{k}
$$

Substituting this into the expression for $\hat{A}$, we get

$$
\begin{aligned}
\hat{A}(\hat{\rho}) & =\sum_{j}\left(\sum_{k} U_{j k} \hat{b}_{k}\right) \hat{\rho}\left(\sum_{l} U_{j l} \hat{b}_{l}\right)^{\dagger} \\
& =\sum_{j k l} U_{j k} U_{j l}^{*} \hat{b}_{k} \hat{\rho} \hat{b}_{l}^{\dagger} \\
& =\sum_{k l} \delta_{k l} \hat{b}_{k} \hat{\rho} \hat{b}_{l}^{\dagger} \\
& =\sum_{k} \hat{b}_{k} \hat{\rho} \hat{b}_{k}^{\dagger} .
\end{aligned}
$$

In other words, the superoperator $\hat{A}$ has the same expression in terms of $\hat{b}_{k}$ as it does in terms of $\hat{a}_{k}$. The same identity is valid for $\hat{N}$. Therefore, equal attenuation of all the modes before the interferometer is exactly equivalent to the same attenuation after the interferometer.

Our scheme is therefore equivalent to that shown in Fig. 3. Because the attenuators act independently on each mode, we can without loss of generality assume the loss on mode 1 occurs after measurement of other modes. We call the state of mode 1 conditioned on the desired measurement result, but before the attenuation, $\hat{\rho}_{\text {out }}^{\prime}$. The diagonal elements of density matrices $\hat{\rho}_{\text {olut }}^{\prime}$ and $\hat{\rho}_{\text {out }}$ are related by the Bernoulli transformation [14]:

$$
\left\langle n\left|\hat{\rho}_{\text {out }}\right| n\right\rangle=\sum_{m=n}^{\infty} p_{\max }^{n}\left(1-p_{\text {max }}\right)^{m-n}\left(\begin{array}{c}
m \\
n
\end{array}\right)\left\langle m\left|\hat{\rho}_{\text {out }}^{\prime}\right| m\right\rangle .
$$

According to the above equation, if we require that state $\hat{\rho}_{\text {out }}$ contains no photon number components with $n>1$, the same must be true for $\hat{\rho}_{\text {out }}^{\prime}$. The probabilities of 1 photon components in these states are then related as

$$
X \equiv\left\langle 1\left|\hat{\rho}_{\text {out }}\right| 1\right\rangle=p_{\max }\left\langle 1\left|\hat{\rho}_{\text {out }}^{\prime}\right| 1\right\rangle .
$$

That is, the probability of 1 photon after the loss cannot be larger than $p_{\max }$. This solves the first of the two conjectures formulated in the beginning of this paper.
We solve the other conjecture in two steps. First, we show that it is impossible to increase the probability of a single photon above $p_{\max }$ for $p_{\max } \geq 1 / 2$, allowing multiphoton components in the output. For $n=1$, Eq. (7) takes the form

$$
X=p_{\max } \sum_{m=1}^{\infty}\left(1-p_{\max }\right)^{m-1} m\left\langle m\left|\hat{\rho}_{\text {out }}^{\prime}\right| m\right\rangle .
$$

For $p_{\max } \geq 1 / 2,\left(1-p_{\max }\right)^{m-1} m \leq 1$, so

$$
X \leq p_{\max } \sum_{m=1}^{\infty}\left\langle m\left|\hat{\rho}_{\text {out }}^{\prime}\right| m\right\rangle \leq p_{\max }
$$

as required.

Second, we show that one cannot construct a scheme that generates the output state with the single-photon probability $X>1 / 2$ from inefficient SPs with $p_{\max }<$ $1 / 2$. Indeed, suppose such a scheme exists. But then we could also use it with inefficient SPs of any efficiency $Y$, such that $1 / 2<Y<X$, by first attenuating them. This would however lead to efficiency improvement from $Y$ to $X$, which, as we just showed, is impossible.

The generality of our method also enables us to derive new results that were not anticipated in previous work. In particular, we can define a more general form of the efficiency that may be used for states with multiphoton components. We do this by again considering an initial state followed by a loss channel. We can define $E(\hat{\rho})$ by

$$
E(\hat{\rho}) \equiv \min \left\{p \mid \exists \hat{\rho}^{0} \geq 0: \mathcal{E}_{p}\left(\hat{\rho}^{0}\right)=\hat{\rho}\right\},
$$

where $\mathcal{E}_{p}$ indicates the loss channel with transmission probability $p$. That is, the efficiency is the minimum transmission probability for a loss channel such that $\hat{\rho}$ can be obtained from a valid quantum state (i.e., with positive semidefinite density operator).

This definition may be used for both single-mode and multimode states. In the case where the state is a tensor product of states in the individual modes, $\hat{\rho}=\otimes_{k} \hat{\rho}_{k}$, the generalized efficiency is the maximum of that for the individual modes: $E(\hat{\rho})=p_{\max } \equiv \max _{k}\left(p_{k}\right)$, where $p_{k}=$ $E\left(\hat{\rho}_{k}\right)$. To show this, let us define for each mode the state $\hat{\rho}_{k}^{0}$, such that $\mathcal{E}_{p_{k}}\left(\hat{\rho}_{k}^{0}\right)=\hat{\rho}_{k}$. Then for the states $\hat{\rho}_{k}^{\prime}=\mathcal{E}_{p_{k} / p_{\max }}\left(\hat{\rho}_{k}^{0}\right)$, we have

$$
\mathcal{E}_{p_{\max }}\left(\otimes_{k} \hat{\rho}_{k}^{\prime}\right)=\otimes_{k} \hat{\rho}_{k}=\hat{\rho},
$$

which means, according to definition (11), that $E(\hat{\rho}) \leq$ $p_{\max }$. On the other hand, for any state $\hat{\rho}_{0}$ satisfying $\mathcal{E}_{E(\hat{\rho})}\left(\hat{\rho}^{0}\right)=\hat{\rho}$, tracing over all modes except $k$ gives a state $\hat{\rho}_{k}^{\prime \prime}$ such that $\mathcal{E}_{E(\hat{\rho})}\left(\hat{\rho}_{k}^{\prime \prime}\right)=\hat{\rho}_{k}$. Comparing this with Eq. (11), we obtain $\forall k E(\hat{\rho}) \geq p_{k}$, and thus $E(\hat{\rho})=p_{\max }$.

By applying the procedure of commuting the loss channel with the interferometer, we find that the generalized efficiency cannot be increased under $L O$ processing. This general result includes the above no-go results for improving the ISPS efficiency as particular cases. This is 
because the generalized efficiency of an ISPS is identical to that defined by Eq. (1), and for a set of ISPSs, the generalized efficiency is the maximum of that for the individual single photon sources.

A subtlety is that, with multiphoton components, the generalized efficiency does not necessarily equal the single-photon probability. For coherent states, for example, the generalized efficiency is zero, even though the single-photon efficiency is nonzero. For the two-photon Fock state the relation is opposite. In cases where the single-photon probability of an ISPS is increased through LO processing (as in Refs. [6, 8]), the generalized efficiency is not increased because multiphoton components are introduced.

An important feature of the generalized efficiency is that, if it is possible to interconvert between two states using LO processing and postselection, then they must have the same generalized efficiency. For example, consider the case of partially mixed states of zero and one photon,

$$
\hat{\rho}=(1-p)|0\rangle\langle 0|+q| 0\rangle\left\langle 1\left|+q^{*}\right| 1\right\rangle\langle 0|+p| 1\rangle\langle 1| .
$$

It is shown in Ref. [9] that these states can be interconverted, using LO processing and conditional measurements, with ISPSs of efficiency

$$
E^{\prime}(\hat{\rho})=p /\left(1-|q|^{2} / p\right)
$$

Hence the generalized efficiency $E(\hat{\rho})$ of the partially mixed state (13) must be equal to $E^{\prime}(\hat{\rho})$ [15].

Thus we find that the technique of commuting an equal-loss channel with the interferometer enables us to resolve two long-standing problems from previous work. We find that the efficiency of single-photon sources cannot be increased using linear optics and destructive conditional measurements if it is required that the generated state contain no multiphoton components. Even if this restriction is lifted, it is not possible to increase the single photon probability if $p_{\max } \geq 1 / 2$. We formulate a general definition of the quantum efficiency of an optical state which cannot increase under LO processing. These results place strong performance bounds on all linear optical quantum processing schemes.
One possibility that our results do not rule out is catalytic improvement of photon sources. That is, if there is one source with very high efficiency, it might be possible to use this source to improve the efficiency of multiple sources. This is a topic for future study.

This work has been supported by NSERC, AIF, CIFAR and Quantum Works. We thank B. C. Sanders for inspiring discussions.

[1] G. S. Buller and R. J. Collins, Meas. Sci. Technol. 21, 012002 (2010).

[2] Ph. Grangier, B. Sanders, and J. Vuckovic, eds., "Focus on Single Photons on Demand," New. J. Phys. 6 (2004).

[3] A. Politi, J. Matthews, M. G. Thompson, J. L. O'Brien, IEEE J. Sel. Top. Quantum Electron. 15, 1673 (2009).

[4] E. Knill, R. Laflamme, and G. J. Milburn, Nature 409, 46 (2001).

[5] C. M. Dawson, H. L. Haselgrove, and M. A. Nielsen, Phys. Rev. Lett. 96, 020501 (2006).

[6] D. W. Berry, S. Scheel, C. R. Myers, B. C. Sanders, P. L. Knight, and R. Laflamme, New J. Phys. 6, 93 (2004).

[7] D. W. Berry, A. I. Lvovsky, and B. C. Sanders, J. Opt. Soc. Am. B 24, 189 (2007).

[8] D. W. Berry, S. Scheel, B. C. Sanders, and P. L. Knight, Phys. Rev. A 69, 031806(R) (2004).

[9] D. W. Berry, A. I. Lvovsky, and B. C. Sanders, Opt. Lett. 31, 107 (2006).

[10] N. Imoto, H. A. Haus, and Y. Yamamoto, Phys. Rev. A 32, 2287 (1985).

[11] P. Kok, H. Lee, and J. P. Dowling, Phys. Rev. A 66, 063814 (2002)

[12] M. Varnava, D. E. Browne, and T. Rudolph, quant-ph/0702044 (2007).

[13] D. F. Walls and G. J. Milburn, Quantum Optics (Springer, Berlin-Heidelberg, 1994).

[14] U. Leonhardt, Measuring the Quantum State of Light (Cambridge University Press, Cambridge, 1997).

[15] In fact, Ref. [9] proposed the quantity $E^{\prime}(\hat{\rho})$ as the "generalized efficiency" of the partially mixed state (13). The new generalized efficiency we define here subsumes that earlier definition. 